\title{
Bosonization for 2D Interacting Fermion Systems: Non-Fermi Liquid Behavior
}

\author{
Y. Yu, Y. M. Li and N. d'Ambrumenil \\ Department of Physics, University of Warwick, \\ Coventry, CV4 7AL, U. K.
}

\begin{abstract}
Non-Fermi liquid behavior is found for the first time in a two-dimensional (2D) system with non-singular interactions using Haldane's bosonization scheme. The bosonized system is solved exactly by a generalized Bogoliubov transformation. The fermion momentum distribution, calculated using a generalized Mattis-Lieb technique, exhibits a non-universal power law in the vicinity of the Fermi surface for intermediate interaction strengths.
\end{abstract}


In recent years, there has been great interest in possible non-Fermi-liquid-like behavior in the ground state of two-dimensional (2D) strongly correlated fermionic systems [1]. It is well-known that in one dimension the ground state of a fermionic system with arbitrarily weak interactions is not a Fermi liquid but a state usually known as a Luttinger liquid [2]. For the 2D case, many studies have suggested that, in the limit of weak interactions, this behavior does not appear [3]. Studies of systems with a small number of particles also suggest Fermi liquid behavior in 2D [4]. Although Khveshchenko et al have found nonFermi-liquid behavior in 2D, they worked only with a singular long-range current-current interaction [5].

The one-dimensional Luttinger liquid solution can be obtained by a bosonization procedure [2]. Haldane has recently generalized this procedure to solve 2D models [6] and his method has been further developed in [5]. In this paper, we show using this procedure that, even for non-singular short-range interactions, 2D systems may show non-Fermi-liquid behavior if the interaction is strong enough. However, in the limit of weak interactions it is difficult to distinguish between a Fermi liquid and a non-Fermi liquid.

We diagonalize the bosonized model exactly using a generalized Bogoliubov transformation. The fermion momentum distribution $n(\mathbf{p})$ at zero temperature is then calculated using a generalization to higher dimensions of the method used by Mattis and Lieb [7] in 1D. The power law obtained in 1D for $n(p)$ near $p_{F}, n(p) \sim\left(p-p_{F}\right)^{\delta}$, also emerges in 2D. The exponents, $\delta$, can be calculated numerically. We look in particular at the case of a $\delta$-function interaction and find that the variation of $\delta$ with coupling constant is similar to that found in $1 \mathrm{D}$, although $\delta$ is always smaller in 2D than $1 \mathrm{D}$ for comparable values of the coupling constants. 
We begin with a brief description of Haldane's procedure. Consider the fermionic system with the spectrum $\epsilon(\mathbf{p})=v\left(\left|p_{x}\right|+\left|p_{y}\right|\right)$. The spectrum has 4 different branches with one in each quadrant of the p-plane. One constructs 4 different density operators $B_{\alpha}(\mathbf{q})=\sum c_{\alpha, \mathbf{p}+\mathbf{q}}^{\dagger} c_{\alpha \mathbf{p}}$ where $\alpha$ is the quadrant index and $\mathbf{p}$ and $\mathbf{p}+\mathbf{q}$ lie in the $\alpha$-th quadrant. It is easy to show that those operators obey the commutation relations

$$
\left[B_{\alpha}(\mathbf{q}), B_{\beta}\left(-\mathbf{q}^{\prime}\right)\right]=-\delta_{\alpha \beta} \delta_{\mathbf{q}, \mathbf{q}^{\prime}} \frac{\Lambda \Omega}{(2 \pi)^{2}} \mathbf{n}_{\alpha} \cdot \mathbf{q}
$$

where $\Lambda$ and $\mathbf{n}_{\alpha}$ are the length and normal vector of the Fermi surface in the $\alpha$-th quadrant. $\Omega$ denotes the volume of the system. In terms of the commutation relations between the kinetic energy and $B_{\alpha}(\mathbf{q})$, the total bosonized Hamiltonian reads

$$
H=\frac{(2 \pi)^{2} v}{\Lambda \Omega} \sum_{\mathbf{q}, \alpha, \beta}\left(\delta_{\alpha \beta}+\frac{\Lambda \Gamma_{\alpha \beta}(\mathbf{q})}{v}\right) B_{\alpha}(\mathbf{q}) B_{\beta}(-\mathbf{q}),
$$

if the fermionic interaction has the form $\frac{1}{\Omega} \sum_{\mathbf{p}, \mathbf{p}^{\prime}, \mathbf{q}} \Gamma\left(\mathbf{p}, \mathbf{p}^{\prime}, \mathbf{q}\right) c_{\mathbf{p}+\mathbf{q}}^{\dagger} c_{\mathbf{p}^{\prime}-\mathbf{q}}^{\dagger} c_{\mathbf{p}^{\prime}} c_{\mathbf{p}}$. Since the definition of the $B_{\alpha}(\mathbf{q})$ excludes contribution to the density operators in which $\mathbf{p}$ and $\mathbf{p}+\mathbf{q}$ are in different quadrants, certain scattering terms are not included in the bosonized Hamiltonian. These describe the so-called around-the-corner (ATC) scattering. In the limit $q \rightarrow 0$ the contribution of ATC scattering vanishes as $q / \Lambda$. We will return to this question later.

Haldane has pointed out that the $2 \mathrm{D}$ system described by (2) will converge to the Fermi liquid fixed point in the limit $\Lambda \rightarrow 0$ for non-singular interactions. Haldane's observation is obviously correct when the summation over $\alpha, \beta$ is over four quadrants. However, if the summation over $\alpha$ and $\beta$ is taken over an infinite number of sectors instead of four quadrants, as $\Lambda \rightarrow 0$, the situation may change as we shall see below.

We consider an interacting fermion model described by the Hamiltonian

$$
H=\sum_{\mathbf{p}, s} \epsilon(\mathbf{p}) c_{\mathbf{p} s}^{\dagger} c_{\mathbf{p} s}+\frac{(2 \pi)^{2}}{\Omega} \sum_{\mathbf{q}} V(q) \sum_{\mathbf{p}, \mathbf{p}^{\prime}, s, s^{\prime}} c_{\mathbf{p}+\mathbf{q}, s}^{\dagger} c_{\mathbf{p}^{\prime}-\mathbf{q}, s^{\prime}}^{\dagger} c_{\mathbf{p}^{\prime} s^{\prime}} c_{\mathbf{p} s}
$$


where Planck's constant $\hbar$, the speed of light $c$ and lattice length $a$ have been set to unity. We assume the spectrum $\epsilon(\mathbf{p})=v\left(|\mathbf{p}|-p_{F}\right)$ where $p_{F}$ is the Fermi momentum. Thus the Fermi surface is a circle with radius $p_{F}$. Following Khveshchenko et al [5], we divide this circular Fermi surface into an $M$-sided polygon $(M \rightarrow \infty)$, whose side length is $\Lambda$ with $\Lambda \ll p_{F}$. The whole momentum space is thus divided into $M$ sectors. The $\alpha$-th sector, $T_{\alpha}$, is defined by the direction of the unit vector $\mathbf{n}_{\alpha}$. In order to understand the low energy behavior of the system, we only consider a shell of width $L(L \sim \Lambda)$ around the Fermi surface. The bosonic operators are defined as $B_{\alpha s}(\mathbf{q})=\sum_{\mathbf{p} \in \Lambda_{\alpha}, \mathbf{p}+\mathbf{q} \in T_{\alpha}} c_{\alpha, \mathbf{p}+\mathbf{q}, s}^{\dagger} c_{\alpha \mathbf{p} s}$, where $\alpha$ runs from 0 to $M-1 . \Lambda_{\alpha}$ is the area $L \times \Lambda$ and centered at $\mathbf{p}_{F}^{(\alpha)}$, the Fermi momentum in the $\alpha$-th direction. The bosonic operators satisfy the commutation relations of Eq.(1).

The kinetic energy can be written in terms of the $B_{\alpha s}(\mathbf{q})[5]$

$$
H_{k i n} \approx \frac{(2 \pi)^{2} v}{\Lambda \Omega} \sum_{\mathbf{q}, \alpha, s} B_{\alpha s}(\mathbf{q}) B_{\alpha s}(-\mathbf{q})+O\left(q^{2}\right)
$$

The interaction is bosonized to give

$$
\begin{aligned}
H_{V} & =\frac{(2 \pi)^{2}}{\Omega} \sum_{\mathbf{q}, \alpha, s} V^{\prime}(q) B_{\alpha s}(\mathbf{q}) B_{\alpha s}(-\mathbf{q})+V(q) B_{\alpha s}(\mathbf{q}) B_{\alpha,-s}(-\mathbf{q}) \\
& +\frac{(2 \pi)^{2}}{\Omega} \sum_{s, s^{\prime}, \alpha \neq \beta} V(\mathbf{q}) B_{\alpha s}(\mathbf{q}) B_{\beta s^{\prime}}(-\mathbf{q})
\end{aligned}
$$

Here $V^{\prime}(q)=V(q)-V_{1}(q)$ with $V(q)$ corresponding to the contribution from direct scattering and $V_{1}(q)$ to that from exchange scattering. For a $\delta$-function interaction, $V^{\prime}(q)$ vanishes, as required by Pauli's principle [8]. In Eq.(3a), we have restricted the momenta $\mathbf{p}$ and $\mathbf{p}+\mathbf{q}$ included in the interaction term of (3) to $\Lambda_{\alpha}$ and $T_{\alpha}$, respectively, as done in refs. $[6,5]$.

We now solve the bosonized model described by $H=H_{k i n}+H_{V}$. The commutation relations for the $B_{\alpha s}(\mathbf{q})$ allow creation and annihilation operators to be defined as follows. 
For $\mathbf{n}_{\alpha} \cdot \mathbf{q}>0$

$$
\begin{aligned}
& B_{\alpha s}(\mathbf{q})=\sqrt{\frac{\Lambda \Omega}{(2 \pi)^{2}}} \omega_{q, \alpha}^{1 / 2} a_{\alpha s}^{\dagger}(-\mathbf{q}), \\
& B_{\alpha s}(-\mathbf{q})=\sqrt{\frac{\Lambda \Omega}{(2 \pi)^{2}}} \omega_{q, \alpha}^{1 / 2} a_{\alpha s}(-\mathbf{q}),
\end{aligned}
$$

where $\omega_{q, \alpha}=\left|\mathbf{n}_{\alpha} \cdot \mathbf{q}\right|$. For $\mathbf{n}_{\alpha} \cdot \mathbf{q}<0, a_{\alpha s}^{\dagger}(-\mathbf{q})$ and $a_{\alpha s}(-\mathbf{q})$ are replaced by $a_{\alpha s}(\mathbf{q})$ and $a_{\alpha s}^{\dagger}(\mathbf{q})$ respectively. The operators $a_{\alpha s}( \pm \mathbf{q})$ obey the canonical commutation relations $\left[a_{\alpha s}( \pm \mathbf{q}), a_{\beta s^{\prime}}^{\dagger}\left( \pm \mathbf{q}^{\prime}\right)\right]=\delta_{\alpha \beta} \delta_{\mathbf{q}, \mathbf{q}^{\prime}} \delta_{s s^{\prime}}$. Again we divide the $\mathbf{q}$-plane into $M$ regions. Each of them is the region between $\mathbf{n}_{\alpha}$ and $\mathbf{n}_{\alpha+1}$, and is denoted by $D_{\alpha}$. Without losing generality, we consider $M=4 N$ for convenience. Using $\omega_{\mathbf{q}, \alpha}=\omega_{\mathbf{q}, \alpha+2 N}=\omega_{\mathbf{q}, \alpha+4 N}$, the bosonized Hamiltonian can now be rewritten as

$$
H=2 \sum_{n=0}^{2 N-1} \sum_{\mathbf{q} \in D_{n}} \sum_{s, s^{\prime}} \mathcal{A}_{n, s, \mathbf{q}^{\dagger}}^{\dagger} \Omega_{s, s^{\prime}, \mathbf{q}} \mathcal{A}_{n, s^{\prime}, \mathbf{q}}
$$

For computational convenience, we organize the $4 N$-component vectors, $\mathcal{A}_{n, s}^{\dagger}$, so that the first $N$ and last $N$ components are the creation operators. For example, we would have for the cases $n=0$ and $n=1$ :

$$
\begin{aligned}
& \mathcal{A}_{0, s}^{\dagger}=\left[a_{0 s}^{\dagger}, \ldots, a_{N-1, s}^{\dagger}, a_{N s}, \ldots, a_{3 N-1, s}, a_{3 N, s}^{\dagger}, \ldots, a_{4 N-1, s}^{\dagger}\right] \\
& \mathcal{A}_{1, s}^{\dagger}=\left[a_{0, s}^{\dagger}, \ldots, a_{N-1, s}^{\dagger}, a_{3 N, s}, a_{N+1, s}, \ldots, a_{3 n-1, s}, a_{N s}^{\dagger}, a_{3 N+1, s}^{\dagger}, \ldots, a_{4 N-1, s}^{\dagger}\right],
\end{aligned}
$$

and so on. Here $a_{\alpha, s}^{\dagger} \equiv a_{\alpha, s}^{\dagger}\left(-\mathbf{q}_{n}\right)$ and $a_{\alpha, s} \equiv a_{\alpha, s}\left(\mathbf{q}_{n}\right)$ and $\mathbf{q}_{n}$ denotes $\mathbf{q} \in D_{n}$. The elements of the matrix $\Omega_{s, s^{\prime}}$ are given by

$$
\begin{array}{ll}
\Omega_{\alpha \beta, s s^{\prime}}(\mathbf{q})=\left[\left(v+\Lambda V^{\prime}(q)\right) \delta_{s s^{\prime}}+\Lambda V(q) \delta_{s,-s^{\prime}}\right] \omega_{\mathbf{q}, \alpha}, & \text { for } \alpha=\beta, \\
\Omega_{\alpha \beta, s s^{\prime}}(\mathbf{q})=\Lambda V(q) \omega_{\mathbf{q}, \alpha}^{1 / 2} \omega_{\mathbf{q}, \beta}^{1 / 2}, & \text { for } \alpha \neq \beta .
\end{array}
$$

We introduce the charge density operators, $R_{\alpha, \mathbf{q}}$, and spin density operators, $S_{\alpha, \mathbf{q}}$, via the relation $a_{\alpha}( \pm \mathbf{q})=\frac{1}{\sqrt{2}}\left(R_{\alpha \mathbf{q}}+s S_{\alpha \mathbf{q}}\right)$. The Hamiltonian (6) can therefore be expressed 
as $H=H_{\sigma}+H_{\rho}$,

$$
\begin{aligned}
& H_{\sigma}=\sum_{n, D_{n}, \alpha} S_{\alpha, \mathbf{q}_{n}}^{\dagger} \tilde{\Omega}_{\alpha \alpha, \mathbf{q}_{n}}^{\sigma} S_{\alpha, \mathbf{q}_{n}}, \\
& H_{\rho}=\sum_{n, D_{n}, \alpha, \beta} \Gamma_{\alpha, \mathbf{q}_{n}}^{\dagger} \tilde{\Omega}_{\alpha \beta, \mathbf{q}_{n}}^{\rho} \Gamma_{\beta, \mathbf{q}_{n}},
\end{aligned}
$$

where $\tilde{\Omega}_{\alpha \alpha, \mathbf{q}}^{\rho, \sigma}=\left(v+\Lambda V^{\prime}(q) \pm \Lambda V(q)\right) \omega_{\alpha, \mathbf{q}}$ with $\rho$ corresponding to ' + ' and $\sigma$ to '-', and $\tilde{\Omega}_{\alpha \beta, \mathbf{q}}^{\rho}=2 \Omega_{\alpha \beta, s s^{\prime}}$ for $\alpha \neq \beta$. The vectors $\Gamma$ correspond to vectors $\mathcal{A}$ in (7) with $a$ 's replaecd by $R$ 's.

The spin density part of the Hamiltonian, $H_{\sigma}$, is already diagonal. There are $4 N$ excitation branches with energies $\tilde{\Omega}_{\alpha \alpha, \mathbf{q}}^{\sigma}$. The branches $S_{\alpha, \mathbf{q}}$ and $S_{\alpha+2 N, \mathbf{q}}$ are degenerate.

We need to diagonalize the density part of the Hamiltonian, $H_{\rho}$. Formally, this can be done by setting up the equation

$$
f(\lambda)=\operatorname{Det}\left|\tilde{\Omega}_{a N+i, b N+j}^{\rho}-\Delta_{a} \lambda \delta_{a b} \delta_{i j}\right|=0,
$$

where $i, j=1, \ldots, N$, and $a, b=0, \ldots, 3$. With our ordering of the $4 N$-components of the vector $\mathcal{A}, \Delta_{a}=+1$ for $a=0,3$ and $\Delta_{a}=-1$ for $a=1,2$. Since $\omega_{\alpha, \mathbf{q}}=\omega_{\alpha+2 N, \mathbf{q}}$, we have $f(\lambda)=f(-\lambda)$. The $4 N$-component 'extended' eigenvectors, $X^{(\mu)}$, satisfy:

$$
\Omega^{\rho} X^{(\mu)}=\lambda_{\mu} \Delta X^{(\mu)}
$$

for each $\lambda_{\mu}$. Here the matrix $\Delta=\left(\Delta_{a} \delta_{a b} \delta_{i j}\right)$. For real $\lambda_{\mu}[9]$, we have the extended normalization and orthogonality relations

$$
X^{-1^{T}} \Delta X^{-1}=\Delta^{\prime}
$$

where $X^{-1}=\left(X^{(0)}, \ldots, X^{(4 N-1)}\right)$ is the inverse of the transformation matrix $X$ and $\Delta^{\prime}=$ $\left(\Delta_{\mu} \delta_{\mu \mu^{\prime}}\right)$ with $\Delta_{\mu}$ equal +1 for $\lambda_{\mu}>0$ and -1 for $\lambda_{\mu}<0$. The transformation matrix, $X=\Delta^{\prime} X^{-1^{T}} \Delta$, can be easily obtained from the orthogonality relations (11). Using 
Eqs.(10) and (11), $H_{\rho}$ is diagonalized to $H_{\rho}=\sum\left|\lambda_{\mu}(\mathbf{q})\right| \theta_{\mu, \mathbf{q}_{n}}^{\dagger} \theta_{\mu, \mathbf{q}_{n}}$, where the $\theta_{\mu, \mathbf{q}_{n}}^{\dagger}$ and $\theta_{\mu, \mathbf{q}_{n}}$ are true bosonic creation and annihilation operators.

To determine the nature of the ground state of the Hamiltonian (8) and, in particular, whether it is a Fermi liquid or not we look at the momentum distribution, $n(\mathbf{p})$. We first extend the method used by Mattis and Lieb in 1D [7] to higher dimensions.

A fermion $\psi_{s}(\mathbf{x})=\sum_{\mathbf{p}} e^{-i \mathbf{p} \cdot \mathbf{x}} c_{\mathbf{p} s}$ is written in terms of operators with momenta restricted to each sector, $\Lambda_{\alpha}$, of the polygonised Fermi surface, i.e. $\psi_{s}(\mathbf{x}) \approx \sum_{\alpha} \psi_{\alpha s}(\mathbf{x})$ where $\psi_{\alpha s}(\mathbf{p})=\sum_{\mathbf{p} \in \Lambda_{\alpha}} e^{-i \mathbf{p} \cdot \mathbf{x}} c_{\alpha, \mathbf{p}, s}$. Then the fermion momentum distribution at zero temperature is

$$
n(\mathbf{p})=\sum_{\alpha} \int d^{2} \mathbf{x} e^{i \mathbf{p} \cdot \mathbf{x}}<G\left|\psi_{\alpha s}^{\dagger}(\mathbf{x}) \psi_{\alpha s}(0)\right| G>
$$

where $\mid G>$ is the vacuum state of the interacting system described by the bosonized Hamiltonian (8).

The ground state, $\mid G>$, can be related to the non-interacting vacuum state $\mid 0>$ by a canonical transformation of the type $\left|G>=e^{-i \Sigma}\right| 0>$, as pointed out by Mattis and Lieb. Under this transformation the fermion operator becomes $\tilde{\psi}_{s}(\mathbf{x})=e^{-i \Sigma} \psi_{s}(\mathbf{x}) e^{i \Sigma}$. We assume $\Sigma$ has the form $i \sum_{n, D_{n}, \alpha, \beta} \Gamma_{\alpha \mathbf{q}_{n}}^{\dagger} A_{\alpha \beta, \mathbf{q}_{n}} \Gamma_{\beta \mathbf{q}_{n}}$. The matrix $A$ can then be determined from $e^{O}=X$ with $O_{\alpha \beta}=\Delta_{\alpha} A_{\alpha \beta}$. As in the $1 \mathrm{D}$ case, the fermion operator in the bosonic representaion is of the form $\psi_{\alpha s}(\mathbf{x})=F_{\alpha s}(\mathbf{x}) e^{J_{\alpha s}(\mathbf{x})}$ where $F(\mathbf{x}) \sim e^{i \mathbf{p}_{F}^{(\alpha)} \cdot \mathbf{x}}$ can be an arbitrary function of $\mathbf{x} . \quad J(\mathbf{x})$ is the current operator and is very analogous to its counterpart in 1D. Dividing $J$ into $J^{\sigma}$ and $J^{\rho}$ relating to spin and charge currents respectively and solving the 'equation of motion' for $f_{\alpha \tau}=e^{i \tau \Sigma} \psi_{\alpha}(\mathbf{x}) e^{-i \tau \Sigma}$ with time ' $\tau$ ' $[7,5]$, one obtains

$$
\tilde{\psi}_{\alpha s}(\mathbf{x})=W_{\alpha}(\mathbf{x}) Y_{\alpha}(\mathbf{x}) \psi_{\alpha s}
$$

Since the elements of the transformation matrix $X$ satisfy $X_{\alpha \beta}=X_{2 N+\alpha, 2 N+\beta}$, the oper- 
ators $W_{\alpha}$ and $Y_{\alpha}$, in the cases for $\alpha=i=0, \ldots, N-1$, are given by

$$
\begin{aligned}
W_{i}(\mathbf{x}) & =\exp \frac{2 \pi}{\sqrt{2 \Lambda \Omega}}\left\{\sum_{D_{ \pm}} \frac{X_{i i}-1}{\omega_{i \mathbf{q}}^{1 / 2}}\left[e^{ \pm i \mathbf{q} \cdot \mathbf{x}} R_{i \mathbf{q}}-e^{\mp i \mathbf{q} \cdot \mathbf{x}} R_{i \mathbf{q}}^{\dagger}\right]\right\} \\
Y_{i}(\mathbf{x}) & =\exp \frac{2 \pi}{\sqrt{2 \Lambda \Omega}} \sum_{\beta \neq i}\left\{\sum_{D_{ \pm}} \frac{X_{i \beta}}{\omega_{i \mathbf{q}}^{1 / 2}}\left[e^{ \pm i \mathbf{q} \cdot \mathbf{x}} \Gamma_{\beta \mathbf{q}}-e^{\mp i \mathbf{q} \cdot \mathbf{x}} \Gamma_{\beta \mathbf{q}}^{\dagger}\right]\right\}
\end{aligned}
$$

where $D_{+}=\left\{D_{0}, \ldots, D_{N-1+i}\right\}$ and $D_{-}=\left\{D_{N+i}, \ldots, D_{2 N-1}\right\}$. Similar relations follow for $\alpha=N, \ldots, 4 N-1$. These results, combined with the orthogonality relations (11), lead, after some algebra similar to that in ref.[5], to

$$
<G\left|\psi_{\alpha s}^{\dagger}(\mathbf{x}) \psi_{\alpha s}(0)\right| G>=\exp \left\{-Q_{\alpha}(\mathbf{x})\right\}<0\left|\psi_{\alpha s}^{\dagger}(\mathbf{x}) \psi_{\alpha s}(0)\right| 0>
$$

where

$$
\begin{aligned}
& Q_{\alpha}(\mathbf{x})= \frac{(2 \pi)^{2}}{\Lambda \Omega} \sum_{\beta, \mathbf{q} \in D_{\beta}} \frac{1}{\omega_{\beta \mathbf{q}}} f_{\alpha}(\mathbf{q})(\cos \mathbf{q} \cdot \mathbf{x}-1), \\
& f_{\alpha}(\mathbf{q})= \begin{cases}\sum_{j=0}^{N-1}\left(X_{\alpha j}^{2}(\mathbf{q})+X_{\alpha, 3 N+j}^{2}(\mathbf{q})\right)-1, & \text { if } \lambda_{\alpha}>0, \\
\sum_{j=0}^{N-1}\left(X_{\alpha, N+j}^{2}(\mathbf{q})+X_{\alpha, 2 N+j}^{2}(\mathbf{q})\right)-1, & \text { if } \lambda_{\alpha}<0 .\end{cases}
\end{aligned}
$$

It is seen from (12) and (15) that the momentum distribution $n(\mathbf{p})$ is given in terms of the quantities $Q_{\alpha}(\mathbf{x})$. Once these are known we can establish the nature of any singularity in $n(\mathbf{p})$ near $p_{F}$. We note that, although the unobservable quantity $f_{\alpha}(\mathbf{q})$ depends on the ordering of $\left\{\lambda_{\alpha}\right\}$ via (16), the physical quantity $n(\mathbf{p})$ does not.

To illustrate the method, we have solved for the $X_{\alpha \mu}$ for the case of a simple $\delta$-function interaction. In this case $V(\mathbf{q})=U$ and $V^{\prime}(\mathbf{q})=0$ and we can define the dimensionless coupling constant $g=\pi p_{F} U / v$. We denote the component of q perpendicular (parallel) to $\mathbf{n}_{\alpha_{0}}$ for any $\alpha_{0}$ as $q_{\perp}\left(q_{\|}\right)$and scale them by $t_{\|}=q_{\|} / L$ and $t_{\perp}=q_{\perp} / \Lambda$. For any given $M, g$ and $\mathbf{t}$, we denote by $f_{\max }(\mathbf{t})$ and $Q_{\max }(\mathbf{x})$ the values of $f_{\alpha}(\mathbf{t})$ and $Q_{\alpha}(\mathbf{x})$ corresponding to the maximum and minimum values of $\lambda_{\alpha}, \pm \lambda_{\max }$. We also order the $\lambda_{\alpha}$ so that $\lambda_{\alpha}=\lambda_{\max }$ for $\alpha=\alpha_{0}$ and $\lambda_{\alpha}=-\lambda_{\max }$ for $\alpha=\alpha_{0}+2 N$. 
We start with some empirical observations on the results we obtain. For small $g$ $(g \leq 0.05)$ we find that $f_{\max }(\mathbf{t})$ depends on $\mathbf{t}$ and is comparable to the other $f_{\alpha}$. However, above $g \sim 0.05$ there is a crossover to a regime in which $f_{\max }(\mathbf{t})$ does not depend on $\mathbf{t}$ and is significantly larger than the other $f_{\alpha}: f_{\alpha} / f_{\max } \sim 10^{-1}$ for $g=0.05$ while $f_{\alpha} / f_{\max } \sim 10^{-3}$ for $g \geq 1.0$. As $f_{\max }$ turns out to correspond to an exponent we denote it by $\delta$. We find that, for any given $g, \delta$ converges to a constant as $M$ increases. This is shown in Fig. 1. When $\delta$ is much larger than the other $f_{\alpha}$, we retain only $Q_{\max }$ and set all other $Q_{\alpha}$ to zero. This allows us to write

$$
\begin{aligned}
n(\mathbf{p}) & \approx \int d^{2} \mathbf{x} \int_{\mathcal{D}_{\alpha_{0}}} d^{2} \mathbf{k} e^{-Q_{\max }(\mathbf{x})}\left[e^{-i(\mathbf{k}-\mathbf{p}) \cdot \mathbf{x}}+e^{i(\mathbf{k}+\mathbf{p}) \cdot \mathbf{x}}\right] \theta\left(|\mathbf{k}|-p_{F}\right) \\
& +\sum_{\alpha \neq \alpha_{0}, \alpha_{0}+2 N} \int_{\mathcal{D}_{\alpha}} d^{2} \mathbf{k} \delta(\mathbf{p}-\mathbf{k}) \theta\left(|\mathbf{k}|-p_{F}\right)
\end{aligned}
$$

where the integration domains, $\mathcal{D}_{\alpha}$, are the sectors corresponding to $D_{\alpha}$ in the full $\mathbf{k}$-space and $<0\left|\psi^{\dagger}(\mathbf{x}) \psi(0)\right| 0>=1 / \Omega \sum_{\mathbf{k}} \theta\left(|\mathbf{k}|-p_{F}\right)$, the free fermion distribution, is used.

We now evaluate $n(\mathbf{p})$ from (17). Since the angle $\alpha_{0}$ can be chosen arbitrarily, it is convenient to assign $\alpha_{0}$ in such a way that it labels the sector containing $\mathbf{p}$. The second term in $(17)$ then vanishes. Since $\mathbf{p} \in \mathcal{D}_{\alpha_{0}}, Q_{\max }(\mathbf{x}) \approx Q_{\max }\left(x_{\|}, 0\right)$ and the integration over $\mathbf{t}$ leaves

$$
Q_{\max }\left(x_{\|}, 0\right)=\delta \int_{0}^{W} \frac{d s_{\|}}{s_{\|}}\left(1-\cos s_{\|}\right),
$$

where $W=L x_{\|} / 2$. For $W \gg 1$, this gives $Q\left(x_{\|}, 0\right)=\delta \ln W^{2}$ (see [5]) and, after integrating over $x_{\|}$in (18), we obtain

$$
n(\mathbf{p})=\text { const. }-c\left(\frac{|\mathbf{p}|-p_{F}}{L}\right) \delta \operatorname{sign}\left(|\mathbf{p}|-p_{F}\right),
$$

with $c=2^{\delta} I(\delta) / \pi \delta$ and $I(\delta)=\int_{0}^{\infty} d s_{\|} s_{\|}^{-\delta} \cos s_{\|}$. The power exponent $f_{\max }=\delta$ is the function of $g$, shown in Fig. 2 .

For the case of intermediate coupling $g \geq 0.05$, where we can obtain the approximate 
form for $n(\mathbf{p})$ given in (19), one can see clearly that the $2 \mathrm{D}$ system is not a Fermi liquid. In Fig. 2 we compare the variation of $\delta$ with $g$ to the function $\delta\left(g_{1}\right)=\frac{1+g_{1}}{\sqrt{1+2 g_{1}}}-1$ for the $1 \mathrm{D}$ case $\left(g_{1}=2 U / v\right)$. For the $2 \mathrm{D}$ case the non-Fermi-liquid exponent is always smaller than in the $1 \mathrm{D}$ case for comparable values of the dimensionless coupling constants.

For weak coupling $g \leq 0.05$, the contributions from $\pm \lambda_{\max }$ are not dominant and contributions from all of $\lambda_{\alpha}$ are comparable. We have noticed that the largest of the $f_{\alpha}$ varies as $10^{-2(n+1)}$ for $g \sim 10^{-n}$. This clearly implies that it will be difficult to distinguish between Fermi-liquid and non-Fermi-liquid-like behaviors. This might be the reason why the non-Fermi-liquid state has not been found for weak interactions using perturbative approaches [3].

We would like to comment on the neglect of the around-the-corner(ATC) scattering. One might expect that in the limit of a large number of sectors $(\Lambda \rightarrow 0)$, ATC scattering should become increasingly important, as more and more states are close to the corner of a sector. However, as our results show that the exponent, $\delta$, is already well-converged for a small number of sectors, we do not believe that neglect of ATC scattering poses serious problems.

Although we have located at the bosonization of the Hamiltonian for a homogeneous two-dimensional system, we end up with a Hamiltonian which resembles that for coupled chains discribed by Wen [10]. Our results differ from those he obtained for coupled chains in one very significant feature. In Wen's case the interchain interactions modify the single chain Luttinger liquid state. In our case the interactions between sectors generate the non-Fermi liquid state. If we switch off this interaction the system reverts back to a Fermi liquid state and not a series of uncoupled 1D Luttinger liquids.

In conclusion, we have discussed a generalization to $2 \mathrm{D}$ of the Tomonaga-Luttinger 
model using Haldane's bosonization procedure. The bosonized system has been solved exactly by a generalized Bogoliubov transformation. The fermion momentum distribution at zero temperature has been calculated using an extension of the Mattis-Lieb technique. We have found that the momentum distribution exhibits a non-universal power law behavior in the vicinity of the Fermi surface for intermediate interaction strengths. It seems to us that this is the first time that Luttinger liquid behavior has been found nonperturbatively for a two-dimensional model with a non-singular interaction.

We are very grateful to D. P. Chu for useful discussions. This work was supported in part by SERC of the United Kingdom under grant No.GR/E/79798 and also by MURST/British Council under grant No.Rom/889/92/47. 


\section{REFERENCES}

[1] P. W. Anderson, Phys. Rev. Lett. 64, 1839 (1990); 65, 2306 (1990); 66, 3226 (1991);67 , 2092 (1992).

[2] F. D. M. Haldane, J. Phys. C 14, 2585 (1981).

[3] J. R. Engelbrecht and M. Randeria, Phys. Rev. Lett. 65, 1032 (1990); 66, 3325 (1991); Phys. Rev. B 45, 12419(1992); J. R. Engelbrecht, M. Randeria and L. Zhang, Phys. Rev. B 45, 10135 (1992); P. A. Bares and X. G. Wen preprint, 1992; C. Castellani, C. Di Castro and W. Metzner, preprint, 1993. R. Shankar, to be published.

[4] M. Fabrisio, A. Parola and E. Tosatti, Phys. Rev. B 44, 1033 (1991).

[5] D. V. Khveshchenko, R. Hlubina and T. M. Rice, preprint, ETH-TH/93-3, 1993, to appear in Phys.Rev. B.

[6] F. D. M. Haldane, Varenna lectures, 1992 and Helv. Phys. Acta. 65, 152 (1992); also see A. Houghton and B. Marston, Brown University Preprint (1992).

[7] D. C. Mattis and E. Lieb, J. Math. Phys. 6 , 304 (1965).

[8] See, for example, G. D. Mahan, Many-Particle Physics, Plenum Press, New York and London, (1990, 1981).

[9] We have checked that for $\delta$-function interaction all of $\lambda_{\mu}$ are real for large $M$.

[10] X. G. Wen, Phys. Rev. B 42,6623 (1990). 


\section{CAPTIONS OF FIGURES}

Figure 1. The exponents $\delta$ as a function of the number of sectors of the polygonised Fermi surface, $M$, for various values of the dimensionless coupling constant $g$. The exponent, $\delta$, characterises the singularity at the Fermi surface in the momentum distribution function, $n(\mathbf{p})$. As $M$ increases, $\delta$ converges to a constant value for each coupling constant $g$.

Figure 2. The $M \rightarrow \infty$ limit for the exponent, $\delta$, as a function of coupling constant $g$. ( $M$ is the number of sectors of the polygonized Fermi surface). We also show the corresponding results for the $1 \mathrm{D}$ case. 